Revista de Comunicación y Salud, 2018, Vol. 8, no 1, pp. 85-97

Editado por Cátedra de Comunicación y Salud

ISSN: 2173-1675

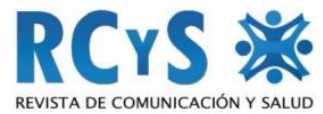

Enviado 14 de agosto de 2018

Aprobado 10 de octubre de 2018

\title{
TECNOLOGÍAS DE LA COMUNICACIÓN Y POSVERDAD: IMPLICACIONES PARA LA GESTIÓN DE LA COMUNICACIÓN HOSPITALARIA
}

\section{Communication Technologies and post-truth: what they imply for the managing hospital communication}

Mario Barquero Cabero ${ }^{1}$, José Rodríguez Terceño y Juan Enrique Gonzálvez Vallés

${ }^{1}$ ESERP Business School de Madrid. España.

"Universidad CEU San Pablo. España.

\begin{abstract}
Resumen
La forma en la que la necesidad y la gestión del conocimiento nos ha llevado a ordenar nuestra visión del conocimiento es mediante la especialización. Una especialización en ocasiones extrema que nos lleva muy a menudo a tener que cruzar especialidades de formas que se nos antojan extrañas, pero que vemos sobre todo en materias de carácter transversal, tendentes a cruzar sus caminos en múltiples campos de experiencia. Una de tales materias es la comunicación, sobre todo a raíz de la implementación de las TIC, que han forzado a instituciones y particulares a ampliar y repensar el modo en el que nos comunicamos, la cantidad de información que transmitimos y como de a menudo la transmitimos. La sociedad se está acostumbrando a un grado de interactividad y de acceso inmediato a la información que obliga a los responsables de comunicación institucional a adaptarse al ritmo o caer en la irrelevancia. Siendo como son, responsables en buena medida de los intereses de la entidad de cara al público, precisan de combinar los valores y la circunstancia de los centros en los que trabajan (en este caso, los sanitarios) con esta nueva realidad. Y una tarea que puede convertirse en bandera y medio de esta labor, es la lucha dentro de su terreno, contra el fenómeno de la posverdad.
\end{abstract}

Palabras clave: posverdad, TIC, gestión hospitalaria, comunicación ética, pacientes.

\begin{abstract}
The way in which the need and management of knowledge has led us to order our vision of knowledge, is through specialization. A sometimes extreme specialization that often leads us to have to cross specialties in ways that seem strange to us, but which we see mainly in matters of a transversal nature, tending to cross their paths in multiple
\end{abstract}

${ }^{1}$ Autor para correspondencia: Mario Barquero Cabero mariobarquero@eserp.com 
fields of expertise. One such issue is communication, especially as a result of the implementation of ICTs, which have forced institutions and individuals to expand and rethink the way in which we communicate, the amount of information that we transmit and how often we transmit it. Society is becoming accustomed to a degree of interactivity and immediate access to information that obliges those responsible for institutional communication to adapt to the accelerating rhythm or fall into irrelevance. Being as they are, largely responsible for the public interests of the entity, they need to combine the values and the circumstances of the centers in which they work (in this case, hospitals and clinics) with this new reality. And a task that can become a banner and a medium for this work, is the struggle within its borders, against the phenomenon of the post-truth.

Keywords: post-truth, ICT's, hospital managing, ethical communication, patients.

\section{Cómo citar el artículo}

Barquero Cabero, M., Rodríguez Terceño, J., Gonzálvez Vallés, J. E. (2018). Tecnologías de la Comunicación y posverdad: implicaciones para la gestión de la Comunicación Hospitalaria. Revista de Comunicación y Salud, 8(1), pp. 85-97. DOI: https://doi.org/10.35669/revistadecomunicacionysalud.2018.8(1).85-97

\section{INTRODUCCIÓN}

La necesidad de comunicación ante la sociedad está cada vez más presente y cada día en más ámbitos distintos. Citando a Caldevilla (2010):

...gracias a Internet y las redes sociales -que son el vivo ejemplo de la interactividad- se puede modificar una conducta, crear nuevos movimientos de opinión, promover manifestaciones, crear grupos de apoyo a causas concretas 0 conseguir crear una moda que genere el consumo de un determinado producto.

Sin embargo, la comunicación aplicada a instituciones no tiene una utilidad exclusivamente corporativa, y en el contexto de las instituciones hospitalarias, resulta necesaria tanto para los sistemas públicos predominantes en Europa, como para los hospitales y clínicas privadas, necesitados de diferenciar su imagen de otras entidades privadas asociadas a entornos normativos y culturales hostiles al concepto de estado del bienestar. Ello hace necesario profesionalizar la comunicación de estas instituciones, dada además la preminencia que los temas relativos a la salud pueden alcanzar en los medios y en las redes en la actual coyuntura mediática y tecnológica, y el margen de reacción cada vez más reducido que las redes sociales y las TIC han impuesto respecto al manejo de crisis comunicativas o al desmentido de noticias falsas. Noticias que, en este ámbito, pueden provocar un daño muy grave a la sociedad, que las instituciones hospitalarias son en parte responsables de frenar. Tampoco puede descartarse el impacto de la nueva cultura de la comunicación, extremadamente participativa y basada en la inmediatez. Ya en 2008 Caldevilla expresaba: 
la recepción de noticias casi al instante de producirse, la facilidad para transmitir información ubicuamente, el intercambio de papeles entre emisor y receptor en la comunicación bidireccional y multipuntual y la posibilidad del libre acceso a las fuentes de información sin intermediarios. Esta novedad derivada de la opulencia comunicativa ¿Supone la necesidad de un nuevo lenguaje para un nuevo (multi)escenario?

Si este impacto es relevante para la comunicación en general, debe valorarse su efecto en este terreno específico. Es por ende relevante plantearnos cómo ha de adaptarse la especificidad de la comunicación de orden sanitario y hospitalario a estas nuevas realidades, que están obligando a un repensamiento generalizado de la comunicación empresarial e institucional.

\section{OBJETIVOS}

Nos proponemos determinar cuáles son los retos asociados a la comunicación corporativa de las entidades específicamente hospitalarias y sanitarias: el efecto concreto que sobre su funcionamiento, gestión y estrategia de comunicación tiene el nuevo escenario post mass-media, y la responsabilidad y campo de acción que pueden tener en los casos en los que el creciente fenómeno de la post-verdad se adentra en el terreno de la medicina y la salud pública. Esperamos encontrar ramificaciones hacia el terreno de la bioética y la publicidad responsable: materias que a su vez pueden generar sinergias de debate en torno a los aspectos económicos de la gestión hospitalaria y sus implicaciones hipocráticas.

\section{METODOLOGÍA}

Procederemos con un proceso inductivo-deductivo destinado a determinar el impacto de la post-verdad y las TIC en el campo de la gestión hospitalaria, previa documentación básica destinada a perfilar los elementos que han de constituir la actividad y la imagen de dichas instituciones. La metodología heurística de análisis de fuentes se apoyará en argumentos de autoridad que aportarán la vertiente cualitativa del estado de la cuestión. Este es estudiado mediante fuentes digitales, con sus implicaciones prácticas y éticas, analizadas como fenómeno de la educación social.

Esta investigación se fundamenta en la importancia y amplitud del tema combinado con la escasa relación percibida entre los ámbitos de la gestión hospitalaria, la medicina y la comunicación. Y en las posibles sinergias que pueden extraerse de aplicar a unas los parámetros de las otras.

\section{DISCUSIÓN}

De acuerdo a la definición más académica (RAE) de lo que implica que una institución sea de carácter hospitalario la función de esta ha de incluir la diagnosis y tratamiento de pacientes, sin menoscabo del apartado investigador y divulgador en el 
ámbito de la medicina. En resumen: Investigación médica, asistencia sanitaria y difusión universitaria.

Asenjo (2002) diferencia estas instituciones en función de:

a) Función -general o especializada-

b) Tipología de los tratados -corta, larga o media estancia para tratamiento-

c) Ámbito demográfico -regional, nacional, internacional...-

d) Nivel de la asistencia -baja o media complejidad, grado tecnológico...-

e) Titularidad pública o privada

Las funciones de todos ellos, cualquiera que sea su clasificación, son similares (McKee, Healy, 2002): tratamiento médico, difusión universitaria y en apoyo interhospitalario. La asunción de tantas tareas por entidades de origen tan diverso fuerza a considerar la inclusión entre el elenco profesional, de personas con formación administrativa y de gestión que ejerzan como coordinadores de dichas funciones. Siguiendo a Mintzberg (2004) una persona involucrada en múltiples tareas mediante técnicas objetivas a toda necesidad administrativa. Para cubrir estas necesidades, la comunicación es una herramienta con mucho potencial. Mucchieli (2005) la define como "el arte de resolver, a través de distintas formas de comunicación interna, los problemas organizacionales comunes y recurrentes que se presentan a todo responsable". Lo que ha llevado a un desarrollo creciente de la actividad comunicativa por parte de las instituciones de carácter hospitalario.

\subsection{Comunicación institucional aplicada a centros hospitalarios}

Son cada vez más los hospitales que se han decidido a incrementar sus esfuerzos en el apartado de la Comunicación Institucional, a menudo teniendo que crear el departamento ad hoc. Este es responsable de definir los elementos básicos que fundamentarán la política de comunicación del centro: su identidad corporativa, su propósito de cara al público, la imagen diferenciadora y su cultura de la comunicación. Todo aquello que posiciona la estrategia de relaciones públicas del hospital y su lugar en un entorno en el cual la comunicación es el arma en la lucha por llamar la atención de un público sobre-estimulado, diferenciándose del resto de entidades. Una realidad que, unida al creciente interés demostrado por la audiencia hacia los temas sanitarios incrementa en sí la visibilidad de los centros hospitalarios.

La identidad de una institución es "un sistema de comunicación que se incorpora a la estrategia global de la empresa y se extiende y está presente en todas sus manifestaciones, producciones, propiedades y actuaciones" (Costa, 2001). El primer paso a este respecto es establecer dicha identidad. El segundo es comunicarla satisfactoriamente al exterior, lográndose con ello efectos, como el de cohesionar eficazmente a la institución, a sus empleados y a los responsables de su comunicación (Van Riel y Fombrun, 2007). 
Tecnologías de la Comunicación y posverdad: implicaciones para la gestión de la Comunicación Hospitalaria

Además de la identidad, el hospital establece su propósito o misión. La meta a partir de la cual su organización se crea: dineraria, social, humanitaria, etc. (Nieto, 2008). Este fin último es definitorio para la situación estratego-comunicativa del hospital, determinando a qué público debe dirigir sus comunicados, priorizar audiencias y escoger la forma en la que aborda sus comunicados.

La visión de la institución hospitalaria es, a juicio de Barret (2003) un nivel de motivación más profundo que una misión. La misión describe los medios, la visión describe el objetivo y establece una declaración cautivadora de lo que la organización intenta conseguir". Mientras que la cultura de la institución lo constituyen las prácticas profesionales e ideas pragmáticas de sus empleados. Promover y, hasta cierto punto controlar esta cultura, requiere dominar la comunicación (Parsons, 2001; Wright, Sparks, O'Hair, 2008). El concepto de control es importante: ha de ser el propio hospital el que administre su imagen, puesto que ya está de por sí muy influido por sus stakeholders o grupos de interés. La imagen es la suma de impresiones individuales, relaciones y material de los mass-media (Van Riel, 1998, p. 95).

Determinar las diferencias y definiciones de estos elementos de la comunicación institucional (identidad, misión, visión, cultura e imagen) es importante para implementar esta de forma adecuada en clínicas y hospitales. Dentro de este, el Dircom y su personal (si lo hay) necesitan también de una completa colaboración por parte del resto de departamentos y de la dirección. (Argenti, 2003, p. 76).

\subsection{Comunicación "On-line" en centros hospitalarios}

Los nuevos desempeños en la investigación médica no vienen sino a complicar aún más el volumen de información del ramo que está disponible en la red. (Reid, 2008). Es así como la red ha influido en la revolución comunicativa del sector. Ello, como viene siendo normal, también ha planteado una serie de nuevos problemas.

Una de ellas viene directamente de la doble necesidad de, por un lado, comunicar de manera efectiva, y por otro, salvaguardar los datos personales y hechos vitales propios de los pacientes (según Rafalski y Mullner, 2003) necesitando compaginar una con otra y con la legislación aplicable a ambos ámbitos y a los centros hospitalarios.

Internet puede igualmente tener un efecto negativo en la percepción que el paciente medio tiene de los centros y el personal médico; del tratamiento, etc. (Fostier, 2005) exponiendo al público a un riesgo grave para la salud general, por ejemplo reemplazando a estos como los principales proveedores de información médica.

Un componente fundamental que puede verse afectado muy fácilmente por la comunicación a escala digital, es su aspecto ético -como señala Mendiz Noguero (2016)- que debe respetarse y estar presente, sobre todo cuando esta se produce en el ámbito de la salud (Guttman, 2003). Este entiende que la red debe servir, en este ámbito, a dos propósitos: auxiliar en la labor profesional sanitaria y colaborar en la recuperación de los pacientes. Un concepto que gana interés de acuerdo a los trabajos

Revista de Comunicación y Salud, 2018, Vol. 8, oㅜ 1, pp. 85-97 
Tecnologías de la Comunicación y posverdad: implicaciones para la gestión de la Comunicación Hospitalaria

al respecto de Agudo-Prado, Pascual-Sevillano y Fombona (2012) y Fombona, Pascual, Iribarren y Pando (2011).

El primer paso en la apuesta por la comunicación hospitalaria pasa por la creación de páginas web que mejoren la fluidez de la relación con el público, principalmente los enfermos y sus familias. Pero también las autoridades competentes y los medios de comunicación. El Dircom y su departamento son los responsables de crear, mantener y alimentar dicha página, acorde a la estrategia de comunicación definida por este, en colaboración con el hospital y las instituciones externas necesarias. En la creación de estos sitios tendremos en cuenta: las consideraciones pedagógicas, los retos de accesibilidad vinculados, la atención individualizada, la omnipresente necesidad de contenido interactivo/interactividad con el público, asociada a un dialogo multilateral.

El enfoque pedagógico prima internet como herramienta para el aprendizaje. Como decían Alonso García y Alonso García (2014):

Los canales de social media cada vez ocupan un lugar más importante, la universidad, los docentes y los alumnos, deben estar preparados para comenzar a utilizar estos canales de comunicación en el ámbito educativo, porque tal como se puede extraer de los resultados las Universidades que tienen mayor número de seguidores poseen una mayor interactuación con sus alumnos y sus publicaciones tienen una mayor viralidad a través de los medios digitales.

El poder pedagógico de la red (Sierra Caballero, 2002) ha llevado a instituciones sanitarias a utilizarla para transmitir al público información de todo tipo sobre hábitos relacionados con la vida saludable, (Campañas de alergias, vacunación, salud alimentaria o cáncer (Moyá Garrido, 2009). En términos de accesibilidad, entendida como accesibilidad web, el amplio espectro de edad que incluye el público de una institución hospitalaria obliga a poner especial atención al uso sencillo de esta (Fisher et al, 2008). La imagen asociada a las páginas de este ámbito consideradas exitosas en su comunicación, es la de fiabilidad y calidad; erudición, y uso sencillo. Desarrollándose desde su creación como satisfactoras de intereses genéricos, a cubrir necesidades más concretas. (Huntington, Nicholas, Williams, 2003).

En este sentido, atendemos necesariamente al criterio de personalización: respondiendo a la creciente necesidad de información individualizada por pacientes (Giustini, 2006). La preeminencia del medio digital como vehículo de comunicación con el público no solo asocia crecientemente la consulta con los casos específicos, sino que también pone el acento en la necesidad de interactividad. Buckley (2007) insiste en que los pacientes llevan ya tiempo dando importancia a la experiencia de atención, y no tanto a la mera información. Lo que nos lleva directamente al concepto de la comunicación on-line, digital o 2.0 a través de redes sociales y otros métodos propios del 2 y 3.0. Citando a Vidales y Rubio (2014):

Como consecuencia de la convergencia tecnológica, que combina formatos, lenguajes y estéticas en las diversas pantallas, se abren nuevos escenarios de 
Tecnologías de la Comunicación y posverdad: implicaciones para la gestión de la Comunicación Hospitalaria

usos y consumo diferenciado para las nuevas audiencias que modifican su rol tradicional en base a la interactividad con los medios.

Respecto a la comunicación interpersonal, la web creada debe facilitarla entre el profesional y el/los pacientes. Contando para ello con varias herramientas diferentes, que permitan a cada uno contactar por el medio que más se adecúe a su necesidad, conocimiento y circunstancia.

\subsection{Comunicación digital: globalización y posverdad como nuevos retos}

Las realidades de la comunicación actual llevan a la necesaria presencia de los medios hospitalarios en el entorno digital, pero también les atribuyen responsabilidades derivadas que no fueron anticipadas en su momento. Del mismo modo que las cuentas de los cuerpos de seguridad y otras instituciones públicas relacionadas se han convertido sin mandato preciso en fuentes para la rápida confirmación o desmentido de bulos, las entidades hospitalarias juegan un papel similar en aquellos casos que afecten a la salud general. No solo por una cuestión deontológica, sino también de interés propio: en un contexto como el actual en el que se coadyuva el auge de las informaciones falsas con una creciente e irreductible creencia en medios acientíficos de curación. Medios que pueden resultar inocuos, hasta que provocan la omisión de tratamiento necesario.

Un ejemplo lo tenemos en la polémica creciente respecto, precisamente, al tema de la extensión de la creencia en la efectividad de la homeopatía. (OMC, AMM, 2018) Una actividad económica que, cual otras prácticas de la historia, se aprovecha de situaciones personales y familiares de vulnerabilidad, en las que los pacientes se hayan receptivos a cualquier solución por improbable que esta sea. La generalización de ideas asociadas a la post-verdad, como las teorías conspirativas, la desconfianza crónica hacia todo lo que pueda considerarse institucional, o la presentación de los medios de comunicación como partes integrantes de algún tipo de plan destinado a buscar el bien de la plutocracia a costa de la gente, está presente en el mapa comunicativo actual. Por citar el ejemplo más sencillo, podemos verlo en la socorrida fórmula "clickbait" (cebo para clics) que todos hemos leído en alguna ocasión, en la que el titular siempre empieza con un "Los médicos la odian" y que suele ir sucedido por el anuncio de algún supuesto medicamento con propiedades estéticas improbables.

Esta es una imagen que se entiende con facilidad si proviene de un entorno en el que se ha permitido que la imagen de la profesión médica decaiga de manera general: ya sea a causa de malas praxis, o de un enfoque empresarial no concienciado con su responsabilidad civil corporativa (Mira, Carrillo y Lorenzo, 2017). Pero existe el riesgo, sobre todo si estas sociedades son culturalmente influyentes, de que esta percepción se traslade a otros entornos, aunque sea de manera parcial. La naturaleza de las informaciones que pueden ser perjudiciales para la salud pública en Internet, hace que resulten tan difíciles de combatir o más que cualquier otro bulo. Por esta razón, su desmentido no puede dejarse únicamente en manos de las autoridades, cuyos medios 
en las redes no alcanzan más allá de los de cualquier tuitero medianamente bien posicionado. Hace falta más. Hacen falta multitud de fuentes que creen en el espectador sensación de verdad contrastada: en el nuevo ámbito comunicacional, no basta con que la información sea cierta. Además tiene que parecerlo.

Respecto a la proliferación de bulos, existen teorías diversas sobre sus causas: desde los que son creados por grupos interesados, hasta los que son obra de individuos con intereses a veces particulares o meramente ideológicos en su difusión. Se suele pensar que estos usuarios (conocidos popularmente como "troles" en referencia al monstruo mitológico escandinavo, más por su faceta desagradable que por la terrible) actúan por inmadurez, buscando atención. Si bien un estudio más detenido llevado a cabo por Griffiths (2014) apunta a que, psicológicamente, responden a perfiles sádicos, psicópatas y maquiavélicos: "una manifestación del sadismo más cotidiano". (Buckels, Trapnell y Paulhus, 2014). El agente de la posverdad mezcla la manipulación de los hechos y la explotación de la relatividad moral y opinativa nietziana con la tendencia natural de las audiencias a secundar sin cuestionar los mensajes que son afines a sus creencias. El éxito de difusión de la posverdad es consecuencia en muy buena medida, de la negativa de naciones y sociedades a guiarse por la evidencia de los hechos antes que en función de sus emociones: la generalización de este tipo de contenido noticiable hace, más que nunca, que en el actual proceso creativo sea decisiva la formación del receptor y del emisor profesional. Entendido en el contexto del presente artículo, como el community manager o el responsable de la comunicación digital (Kadri y Khomsi, 2017) de la entidad o entidades hospitalarias. Formación no sólo por una forma determinada de estructurar la narración de los hechos, sino también por técnicas que permitan igualar el desigual terreno comunicativo de las redes sociales frente al cinismo digital, y especialmente, por los criterios de selección de las fuentes atendiendo a su veracidad (Caldevilla, 2013): apuntando en última instancia a una mejora cualitativa de la forma en que el ciudadano participa en la comunicación, ahora que cuantitativamente tiene la capacidad de actuar y reaccionar sobre ella a un nivel global, previniendo así la extensión de malos hábitos comunicativos a la comunidad de pacientes. De acuerdo a Mut Camacho (2012):

[...] en el siglo XXI la opinión pública ha cristalizado en un ente supranacional con vida propia. La opinión pública se ha materializado en un movimiento social a escala planetaria, donde jamás la humanidad y su opinión tuvieron tanta presencia. Miles, millones de personas en todos los países, en los últimos años, salen a la calle a manifestarse contra la guerra, contra los gobiernos, contra medidas legislativas, contra la economía, etc. Estas acciones son la concretizaron de la opinión pública latente. Una concretización que va permaneciendo de forma continuada, mostrando al mundo que existen unánimes actitudes frente a un tema social o político o económico. Por otra parte, gracias a las nuevas tecnologías, ahora la opinión pública es capaz de organizarse mejor y más rápidamente, además de anunciarse, y toda esta situación significa el advenimiento de la población mundial al poder político de sus países (y de los demás países), y anuncia una reducción de los poderes estatales mediante la 
Tecnologías de la Comunicación y posverdad: implicaciones para la gestión de la Comunicación Hospitalaria

incidencia directa en los asuntos públicos de la libre discusión de los particulares a escala mundial. (Camacho, 2012, pp. 3 y 4).

Joyanes (2000) habla directamente de la era de la Post-información, y de la necesidad de una preparación tanto tecnológica, como cultural, para afrontar las consecuencias de la aldea global de McLuhan.

Caldevilla (2008) habla del concepto de inteligencia colectiva y de cómo se ha visto potenciado por esta nueva realidad:

La verdadera revolución histórica que supone las TT.II.CC. para el posterior desarrollo colectivo de la humanidad radica en las aportaciones colectivas, en los esfuerzos comunes y, en definitiva, en la unificación de avances en una dirección predeterminada. "La inteligencia colectiva es una forma de inteligencia que surge de la colaboración y concurso de muchos individuos". Tal definición surge de los trabajos de Peter Russell (1983), Tom Atlee (1993), Pierre Lévy (1997), Howard Bloom (1995), Francis Heylighen (1995), Douglas Engelbart, Cliff Joslyn, Ron Dembo, Gottfried Mayer-Kress (2003). (Caldevilla, 2008, 139).

Pero como toda inteligencia, la inteligencia colectiva no es ajena a las emociones, y precisa de información como ladrillos para construir conclusiones. Si la información que se le suministra, en este caso a la inteligencia colectiva general, está adulterada, o si esta se halla "infoxicada" con datos contradictorios y/o saturada de ellos, no es razonable esperar que tome decisiones acordes a su interés o su trasfondo cultural.

Volvemos así al concepto de la relación que ha de existir no solo entre el departamento de comunicación y el resto de departamentos, sino entre los dircom de diversos centros hospitalarios, incluso entre aquellos que nominal o comercialmente compitan entre sí. El primer deber de estos centros respecto de la Responsabilidad social corporativa, es el de, simplemente, cumplir con su función social de manera eficaz. En cumplimiento de esta premisa, lo más eficaz es colaborar y hacer frente común frente a la desinformación sanitaria, combatiendo el injustificado desprestigio de las instituciones sanitarias que esta promueve. La estrategia de comunicación de estas instituciones debe hacer frente a esta realidad y poner en valor al profesional médico y de enfermería como fuente de información técnica y como co-protagonista junto al paciente, de la comunicación en el ámbito hospitalario.

Para ello, el hospital puede recurrir a varias iniciativas, como blogs, redes sociales y las app's (aplicaciones) para teléfonos inteligentes. Ya en 2008 Kovic, Lulic y Brumini anotaron la creciente importancia que tenía la blogosfera médica. Estos autores señalaron como características de este "rincón" de internet el alto nivel formativo (el $71 \%$ había realizado masters y/o doctorado), conocimiento elevado de revistas científicas y vinculación profesional al mundo sanitario. Los blogs se han convertido en una fuente de información para pacientes, quienes conceden una creciente credibilidad a este tipo de medio digital. Los blogs pueden enriquecer la comunicación institucional del hospital. Addams (2010) reconoce su utilidad como herramienta para influir en el 
nivel de educación sanitaria de los pacientes. Los pacientes pueden colaborar en estos blogs, compartiendo sus experiencias, iniciativa que resulta coherente con el protagonismo pretendido del profesional sanitario y el paciente: permitiendo que ambos pueden comunicarse libremente a través del hospital. Estos centros también recurren a las redes para agilizar sus comunicaciones sociales. La mayoría de los usuarios de internet de menos de 30 años se han convertido en usuarios activos de estos medios (Sublet, Spring, Howard, 2011), lo cual ha dado lugar al surgimiento de redes sociales especializadas en salud, entre otras materias. Por ejemplo, PatientsLikeMe o de Tsalud.com, en las que los usuarios con formación médica dan consejos formados, y los pacientes comparten sus experiencias. Suponen una oportunidad comunicacionalmente estratégica para que los hospitales den a su comunicación institucional un cariz más interactivo y cercano.

Para el paciente, la comunicación hospitalaria a través de estos canales puede aportarle otros beneficios menos evidentes. Por ejemplo, los MMORPG's (Massive Multiplayer Online Role Playing Games, o Juegos de Rol Online Multijugador Masivo) como World of Warcraft, Ultima Online, Guild Wars o más genéricamente Second Life o Minecraft pueden ayudar a los pacientes y enfermos a combatir la soledad, la falta de objetivos y el aislamiento, de acuerdo a Kamel Boulos, Hetherington y Wheeler (2007), dándonos una herramienta para tratar uno de los flancos más complicados de la recuperación médica: el anímico.

Pero volviendo a las redes sociales especializadas, estas también facilitan al paciente el gestionar sus datos médicos hasta cierto punto: los resultados de las pruebas médicas, por ejemplo. Algo que Tang y Lee (2009) no tienen totalmente claro que resulte práctico. También debemos tener en cuenta los frentes puramente sanitarios que abren estos nuevos medios, como la "depresión por Facebook" diagnosticada por O’Keeffe y Clarke-Pearson (2011), así como los posibles problemas de aislamiento físico.

\section{CONCLUSIONES}

La clave del futuro de la comunicación TIC o 2.0 en las instituciones hospitalarias pasa por facilitar y agilizar con estas nuevas herramientas, la comunicación entre profesionales y pacientes. El objetivo es múltiple: en primer lugar, incrementar la calidad de la atención personal a cada paciente. Pero también proporcionarle información fiable y acertada sobre sus dolencias y sus inquietudes sanitarias. Esta comunicación tiene que proporcionar información verídica y creíble, generando en el proceso un vínculo entre médico y pacientes similar al que históricamente ha existido con el médico de familia: un vínculo de confianza que ayude a combatir la imagen negativa -real 0 importada- que un individuo o grupo de individuos pueda tener de la profesión médica. De forma que el paciente tenga en el profesional de la medicina una defensa temprana y definitiva ante la desinformación médica y las consecuencias negativas que el 2.0 y el entorno de la Post-verdad pueden tener y tienen para la correcta toma de decisiones por parte del paciente. Para lograrlo, existen múltiples herramientas que permiten convertir a médicos y pacientes en comunidades de internet efectivas, estructuradas en

Revista de Comunicación y Salud, 2018, Vol. 8, oㅜ 1, pp. 85-97 
Tecnologías de la Comunicación y posverdad: implicaciones para la gestión de la Comunicación Hospitalaria

torno a foros, blogs y redes sociales especializadas. Con estos medios y estas posibilidades en la mano, el Dircom puede combinar la visión mercantil con la humana y de difusión para formar al paciente como un servicio más, gratuito y no merecedor de ser considerado valor añadido a los servicios del centro. Implicando a los profesionales de la salud en la comunicación del centro, haciendo completamente complementarias las acciones comunicativas digitales y analógicas. Todo ello en una política de estricta observancia de la ética médica por el bien del paciente, pero también para preservar la imagen corporativa, no como un bien empresarial, sino social.

\section{BIBLIOGRAFÍA}

Adams, S. (2010). Blog-based applications and health information: two case studies that illustrate important questions for consumer health informatics $(\mathrm{CHI})$ research. International Journal of Medical Informatics, 79(6), e89-e96.

Agudo-Prado, M.; Pascual-Sevillano, M. y Fombona, J. (2012). "The use of digital tools among the elderly" Revista Comunicar 20(39), 193-201.

Alonso García, S., Alonso García. M. M. (2014). Las redes sociales en las universidades españolas. Revista de Comunicación Vivat Academia, Marzo, año XVII, 126 pp 54-62. DOI: http://dx.doi.org/10.15178/va.2014.126.54-62

Argenti, P. A. (2003). Corporate communication. Boston: McGraw-Hill/Irwin.

Asenjo, M. A. (2002). Las claves de la gestión hospitalaria. 2ª. ed. Barcelona: Gestión 2000.

Barret, Richard; et al. (2003). Libérer l'âme de l'entreprise: bâtir une organisation visionnaire guidée par les valeurs. Bruxelles: De Boeck.

Buckley, Patrick T. (2007). The complete guide to hospital marketing. Marblehead: HCPro Inc.

Caldevilla Domínguez, D. (2008). El nuevo modelo de comunicación a partir de las redes sociales y las TT.II.CC. Revista de comunicación de la SEECI, 16, 115-145. DOI: http://dx.doi.org/10.15198/seeci.2008.16.115-145

Caldevilla Domínguez, D. (2010). Tipología, uso y consumo de las redes 2.0 en la sociedad digital actual. Documentación de las Ciencias de la Información, 33, 45-68.

Caldevilla Domínguez, D. (2013). Efectos actuales de la "Sobreinformación" y la "Infoxicación" a través de la experiencia de las bitácoras y del proyecto I+D avanza 'Radiofriends'. Revista de comunicación de la SEECI, 30, 34-56. Recuperado de www.seeci.net/revista/index.php/seeci/article/view/7/pdf_21

DOI: http://dx.doi.org/10.15198/seeci.2013.30.34-56

Camacho, Mut (2012). Apuntes sobre la opinión pública a pie de calle. Revista de Comunicación de la SEECI, 28, 1-10. Recuperado de www.seeci.net/revista/index.php/seeci/article/view/111

DOI: https://doi.org/10.15198/seeci.2012.28.1-10

Costa, J. (2001). Imagen corporativa en el siglo XXI. Buenos Aires: La Crujía.

Buckels, E. E., Trapnell, P. D., Paulhus, D. L. (2014). Trolls just want to have fun. Personality and Individual Differences. Recuperado de www.sciencedirect.com/science/article/pii/S0191886914000324

DOI: http://doi.org/10.1016/j.paid.2014.01.016 
Tecnologías de la Comunicación y posverdad: implicaciones para la gestión de la Comunicación Hospitalaria

Fisher, J., Burstein, F., Lynch, K. \& Lazarenko, K. (2008). Usability + usefulness = trust: an exploratory study of Australian health web sites. Internet Researchs, 18(5), 477498.

Fombona, J.; Pascual, M.; Iribarren, J., y Pando, P. (2011). "Transparent Institutions" The journal of Systemics, cybernetics and informatics 9(2) 13-16.

Fostier, Pierrik (2005). L'influence de l'internet sur la communication médecin-patient. En: Richard Pierre, Claude \& Lussier, Marie-Theresse (eds.). La communication professionnelle en santé (p. 693-714). Québec: ERPI.

Giustini, D. (2006). How web 2.0 is changing medicine. British Medical Journal, 333 (7582), 1283-1284.

Griffiths, M. (2014). Adolescent trolling in online environments: A brief overview. Education \& Health. Recuperado en: http://sheu.org.uk/x/eh323mg.pdf

Guttman, N. (2003). Ethics in health communication interventions. En: Thompson, T., Dorsey, A., Parrott, R. \& Miller, K. (eds). Handbook of health communication (p. 651679). Mahwah: Lawrence Erlbaum Associates.

Huntington, P.; Nicholas, D.; \& Williams, P. (2003). Characterising and profiling health web user and site types: going beyond hits. Aslib Proceedings, 55(5/6), 277-289.

Joyanes Aguilar, L. (2000). Cambio tecnológico y nueva sociedad de la información (cibersociedad). Revista de Comunicación Vivat Academia, 16, Junio. Recuperado de www.vivatacademia.net/index.php/vivat/article/view/524 DOI: https://doi.org/10.15178/va.2000.15.1-12

Kamel Boulos, M., Hetherington, L. \& Wheeler, S. (2007). Second life: an overview of the potential of 3-D virtual worlds in medical and health education. Health Information and Libraries Journal, 24, 233-245.

Khadri, B. y Reda Khomsi, B. (2017) La metrópoli cultural y turística: ¿una nueva cara de la globalización? Methaodos. Revista de ciencias sociales, 5(1): 38-47. DOI: http://dx.doi.org/10.17502/m.rcs.v5i1.154

Kovic, I.; Lulic, I. \& Brumini, G. (2008). Examining the medical blogosphere: an online survey of medical bloggers. Journal of Medical Internet Research, 10(3), e28.

McKee, M. \& Healy, J. (Eds.) (2002). The role and function of hospitals. En Hospitals in a changing Europe (p. 59-80). Buckingham: Open University Press.

López Vidales, N., Gómez Rubio, L. (2014). La democratización del proceso comunicativo en radio: los jóvenes prosumidores. Revista de Comunicación Vivat Academia, 126, 31-53. DOI: http://dx.doi.org/10.15178/va.2014.126.31-53

Mendiz Noguero, F. (2018). La representación del menor en la publicidad infantil. De la inocencia a la sexualización. Methaodos. Revista de ciencias sociales, 6(1), 125-137. DOI: http://dx.doi.org/10.17502/m.rcs.v6i1.231

Mintzberg, H. (2004). Managers not MBAs: a hard look at the soft practice of managing and gestion development. London: Prentice Hall, Financial Times.

Mira, J. J.; Carrillo, I. y Lorenzo, S. (2017). Qué hacen los hospitales y la atención primaria para mitigar el impacto social de los eventos adversos graves. Gaceta Sanitaria, 31(2), Marzo-Abril, 150-153.

Moyá Garrido, M. N. (2009). Internet, salud y ciudadanía. Sevilla: Agencia de Evaluación de Tecnologías Sanitarias.

Mucchielli, A. (2005). Information et communication interne: étude des communications: pour de nouveaux audits. Paris: Armand Colin. 
Nieto, A. (2008, 10 de marzo). Comunicación institucional: bases para la evaluación. Ideas tomadas en el curso de doctorado impartido por don Alfonso Nieto en la Facultad de Comunicación de la Universidad de Navarra.

O'Keeffe, Schurgin, G. \& Clarke-Pearson, K. (2011). The impact of social media on children, adolescent and famílies. Pediatrics, 127, 800-804.

Organización Médica Colegial de España y Asociación Médica Mundial (2018). La Asociación Médica Mundial apoya una propuesta de Declaración de la OMC sobre las pseudoterapias. Observatorio OMC contra las Psudociencias, pseudoterapias y las sectas sanitarias. Recuperado de http://www.cgcom.es/sites/default/files//u183/np_pseudoterapias_amm_27_04_18.pdf

Parsons, P. J. (2001). Beyond persuasion: the healthcare manager's guide to strategic communication. Chicago: Health Administration Press.

Rafalski, E. \& Mullner, R. (2003). Ensuring HIPAA compliance using data warehouses for healthcare marketing. Journal of Consumer Marketing, 20(7), 629-633.

Reid, G. (2008). Building an accreditation scheme for health and social care nformation. Health Information on the Internet. Recuperado de https://triggered.clockss.org/ServeContent?url=http://hii.rsmjournals.com\%2Fcontent \%2F61\%2F1\%2F3.abstract\#

Sierra Caballero, F. (2002). Comunicación, educación y desarrollo: apuntes para una historia de la comunicación educativa. Sevilla: Comunicación Social.

Sublet, V.; Spring, C. \& Howard, J. (2011). Does social media improve communication? Evaluating the NIOSH science blog. American Journal of Industrial Medicine, 54(5), 384-394.

Tang, P. \& Lee, T. (2009). Your doctor's office or the internet? Two paths to personal health records. The New England Journal of Medicine, 26. DOI: 10.1056/NEJMp0810264

Van Riel, Cees B. M. (1998). Comunicación corporativa. Madrid: Prentice Hall.

Van Riel, Cees. B. M. \& Fombrun, Charles. J. (2007). Essentials of corporate communication: implementing practices for effective reputation gestion. Abingdon: Routledge.

Wong, R., Tan, J. \& Drossman, D. (2010). Here's my phone number, don't call me: physician accessibility in the cell phone and e-mail era. Digestive Disease and Sciences, 55(3), 662-667.

Wright, K. B., Sparks, L. \& O'Hair, D. (2008). Health communication in the 21st century. Malden: Blackwell. 